\title{
THE HOMOTOPY TYPE OF HYPERPLANE POSETS
}

\author{
PAUL H. EDELMAN ${ }^{1}$ AND JAMES W. WALKER ${ }^{2}$
}

\begin{abstract}
Previously, Edelman had defined a partial order on the regions of a euclidean space dissected by hyperplanes. The goal of this paper is to compute the homotopy type of open intervals in these posets. Techniques from the theory of shellable posets are used.
\end{abstract}

0. Introduction. The goal of this paper is to compute the homotopy type of intervals in the hyperplane posets defined in [Ed]. This generalizes [Ed, Theorem 2.5], and certain results of Björner for weak orderings of Coxeter groups $[\mathbf{B j 2}$, Theorem 6]. (See also [Ed, §IV].) In order to accomplish this we prove in $\S 1$ a fact about the homotopy type of certain filters in CL-shellable posets that triangulate spheres. We assume that the reader is familiar with the work of Björner and Wachs $[\mathbf{B W}]$ on CL-shellable posets and the equivalent notion of recursive atom orderings.

Associated with any poset $P$ is the simplicial complex of chains of $P$, called the order complex of $P$. Throughout this paper, we will abuse language by referring to $P$ having certain topological properties when we actually mean the order complex of $P$. In particular, this applies to the title of our paper.

1. A topological theorem. In this section we prove a theorem about the homotopy type of a certain kind of order filter (up-set) in a CL-shellable poset that triangulates a sphere. This theorem will be applied in $\S 2$ to hyperplane posets. For the definitions and notation of CL-shellable posets and recursive atom orderings, we refer the reader to $[\mathbf{B W}]$. A more basic reference for shellability is $[\mathbf{B j} \mathbf{1}]$.

If $P$ is a poset, let $\hat{P}$ denote the new poset formed by adjoining a new least element $\hat{0}$ and a new greatest element $\hat{1}$. If $z \in P$, define $P_{\geq z}=\{x \in P: x \geq z\}$. For $x, y$ in $P$, let $[x, y]$ be the closed interval $\{z \in P: x \leq z \leq y\}$ and let $(x, y)$ be the open interval $[x, y]-\{x, y\}$.

We begin with two lemmas.

LEMMA 1.1. Suppose that $\hat{P}$ admits a recursive coatom ordering $a_{1}, a_{2}, \ldots, a_{t}$ and $z$ is a noncoatom of $\hat{P}$. Let $a_{i_{1}}, a_{i_{2}}, \ldots, a_{i_{k}}, i_{1}<i_{2}<\cdots<i_{k}$, be the coatoms of $[z, \hat{1}]$. Then $a_{i_{1}}, a_{i_{2}}, \ldots, a_{i_{k}}$ is a recursive coatom ordering of $[z, \hat{1}]$.

PROOF. By the equivalence of recursive atom orderings and CL-labellings [BW, Theorem 3.2], it is equivalent to show that a dual CL-labelling of $\hat{P}$ restricts to a

Received by the editors August 10, 1984. The results were presented at the 91st annual meeting of the American Mathematical Society on January 13, 1985, as part of a special session on algebraic combinatorics.

1980 Mathematics Subject Classification. Primary 06A10, 51M20; Secondary 52A25, 55P10, $57 Q 99$.

Key words and phrases. Dissection by hyperplanes, shellability, homotopy type, Möbius function.

${ }^{1}$ Supported in part by NSF grant MCS-8301089.

${ }^{2}$ Supported in part by NSF grant ISP-8011451. 
dual CL-labelling of $[z, \hat{1}]$. But that is a trivial consequence of the definition of CL-labelling.

The next lemma could be avoided, but we think it is of independent interest. It implies a result of Provan $[\mathbf{P r}$, Theorem 5.4.8] which he proved using techniques from PL topology.

LEMMA 1.2. Suppose that $P$ is a poset that triangulates a sphere. If $a_{1}, a_{2}$, $\ldots, a_{t}$ is a recursive coatom ordering of $\hat{P}$, then so is the reverse ordering $a_{t}, a_{t-1}$, $\ldots, a_{1}$.

ProOF. Let us dualize the problem, so that we can speak of recursive atom orderings rather than recursive coatom orderings. By [BW, Theorem 3.2], a recursive atom ordering of $\hat{P}$ can be used to construct a CL-labelling of $\hat{P}$. In fact, the proof constructs an integer CL-labelling $\lambda$ in which no two consecutive labels on the chains are equal. In each rooted interval $[x, y]_{r}$, the number of unrefinable chains with decreasing labels is the absolute value of the Möbius function $\mu(x, y)$, by a formula of Stanley [Bj1, Theorem 2.7]. But since $P$ triangulates a shellable sphere, the open interval $(x, y)$ also triangulates a sphere so $\mu(x, y)= \pm 1$. Therefore each rooted interval has a unique unrefinable chain with decreasing labels.

Moreover, the unique decreasing chain is lexicographically last among all the chains in the rooted interval. We can prove that by induction on the length of the rooted interval, as follows. Let $\mathcal{C}=\hat{0} \rightarrow x_{1} \rightarrow x_{2} \rightarrow \cdots \rightarrow \hat{1}$ be the lexicographically last chain in $[\hat{0}, \hat{1}]$. Then $x_{1} \rightarrow x_{2} \rightarrow \cdots \rightarrow \hat{\mathbf{1}}$ is the lexicographically last chain in $\left[x_{1}, \hat{\mathbf{1}}\right]$ and hence by induction is decreasing. Since $P$ is a sphere, there is exactly one element $z$ other than $x_{1}$ in $\left(\hat{0}, x_{2}\right)$. Since $C$ is lexicographically last, $\lambda(C, \hat{0}, z)<\lambda\left(C, \hat{0}, x_{1}\right)$. Applying the definition of CL-labelling to $\left[\hat{0}, x_{2}\right]$, it follows that $\lambda\left(C, \hat{0}, x_{1}\right)>\lambda\left(C, x_{1}, x_{2}\right)$. Therefore $C$ is decreasing, as desired.

Let $\lambda^{\prime}$ be a new chain labelling defined by $\lambda^{\prime}(C, x, y)=-\lambda(C, x, y)$. Then $\lambda^{\prime}$ has a unique increasing chain in each rooted interval, and this chain is lexicographically first among all unrefinable chains in the interval. This reversed CL-labelling corresponds to the reverse of the original recursive atom ordering.

We can now prove the main theorem of this section.

THEOREM 1.3. Let $P$ be a poset which triangulates a sphere, and let $a_{1}, a_{2}$, $\ldots, a_{t}$ be a recursive coatom ordering of $\hat{P}$. Let $Q$ be the order ideal (down-set) generated by $a_{1}, a_{2}, \ldots, a_{k}$, where $1 \leq k \leq t-1$. Then the order filter $R=P-Q$ is contractible.

Proof. We proceed by induction on the length of $P$. The theorem is easily seen to be true if $P$ has length 1 or 0 .

Let $S$ be the ideal generated by the maximal elements of $R$. That is, $S$ is generated by $a_{k+1}, \ldots, a_{t}$. By Lemma $1.2, a_{t}, a_{t-1}, \ldots, a_{1}$ is a recursive coatom ordering of $\hat{P}$. Since $a_{t}, a_{t-1}, \ldots, a_{k+1}$ is a proper initial segment of that ordering, $S$ is a shellable triangulation of a ball. In particular, $S$ is contractible.

Consider the inclusion map $j: R \rightarrow S$. If we can show that $j$ induces a homotopy equivalence, then $R$ will be contractible as desired. By a theorem of Quillen $[\mathbf{Q u}, \mathbf{p}$. 103] (see also [Wa, Theorem 2.2]), $j$ induces a homotopy equivalence if the fibers $j^{-1}\left(S_{\geq x}\right)=R \cap S_{\geq x}=R \cap P_{\geq x}$ are contractible for each $x$ in $S$. See Figure 1. 


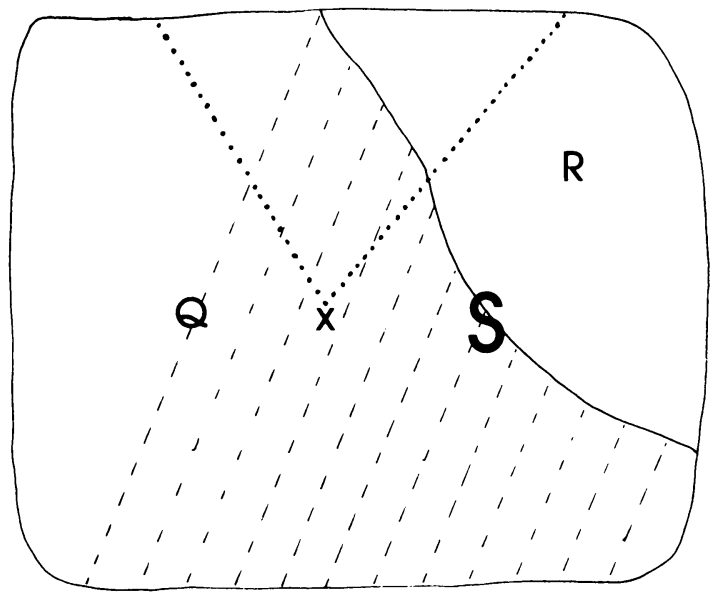

FIGURE 1

If $x \in R$, then $R \cap S_{\geq x}=R_{\geq x}$ is contractible since it has a least element. So assume $x \in S-R$. Then $R \cap P_{\geq x}=P_{\geq x}-Q \cap P_{\geq x}$, and $Q \cap P_{\geq x}$ is nonempty. By Lemma 1.1, the recursive coatom ordering of $\hat{P}$ restricts to a recursive coatom ordering of $[x, \hat{1}]$, and $Q \cap P_{\geq x}$ is an ideal generated by an initial segment of that ordering. Therefore, by inductive hypothesis $R \cap P_{\geq x}$ is contractible. Thus we can apply Quillen's theorem and complete the proof as planned.

2. The homotopy type of hyperplane posets. In this section we apply our Theorem 1.3 to determine the homotopy type of a class of posets related to dissections of $\mathbf{R}^{n}$ by hyperplanes. These posets were first defined in [Ed], and we will use the notation and terminology thereof. We begin by briefly reviewing the definitions.

Let $\mathcal{H}=\left\{H_{1}, H_{2}, \ldots, H_{k}\right\}$ be a set of hyperplanes in $\mathbf{R}^{n}$ whose normal vectors span $\mathbf{R}^{n}$. Then $\mathbf{R}^{n}-\bigcup_{i=1}^{k} H_{i}$ is divided into a set $R$ of open convex $n$-cells called regions.

Let $B$ be a fixed region. For any region $R$ consider the set $S(R)$ defined by $S(R)=\left\{H_{i} \in \mathcal{H}: H_{i}\right.$ separates $R$ from $\left.B\right\}$. The (injective) function $S$ partially orders $R$ by $R_{1} \leq R_{2}$ if and only if $S\left(R_{1}\right) \subseteq S\left(R_{2}\right)$. Let $P(\mathcal{H}, B)$ be the set $R$ with this partial ordering.

The topological closure $\bar{B}$ of $B$ is a convex polytope. The set $B$ of boundary hyperplanes of $B$ is defined by $B=\{H \in \mathcal{H}: H \cap \bar{B}$ is of dimension $n-1\}$. Each hyperplane $H$ in $B$ defines a facet of $\bar{B}, F_{H}^{\prime}$, by $F_{H}^{\prime}=H \cap \bar{B}$. The set of facets of $\bar{B}$ is denoted $\mathcal{F}^{\prime}$. A face of $\bar{B}$ is any intersection of facets of $\bar{B}$. Let $\mathcal{F}$ be the set of faces of $\bar{B}$, other than $\varnothing$ or $\bar{B}$, partially ordered by inclusion. (Excluding $\bar{B}$ and $\varnothing$ is a variation from the notation of [Ed].) If $B$ is bounded, then the boundary of $B$ is a sphere of dimension $n-1$, and $\mathcal{F}$ triangulates that sphere. From $[\mathbf{B M}$ and BW, Theorem 4.3] we know that $\hat{\mathcal{F}}$ admits a recursive coatom ordering.

LEMMA 2.1. If $F \in \mathcal{F}$ then there exists a unique region $R(F)$ such that $S(R(F))=\mathscr{H}(F)$ where $\mathscr{H}(F)=\{H \in \mathscr{H}: H \supseteq F\}$.

PROOF. See [Ed, Lemma 1.2].

A region of the form $R(F)$ for $F$ in $\mathcal{F}$ is called a facial region. 
THEOREM 2.2. If $R \in P(\mathscr{H}, B)$ and $R$ has rank at least 2 , then the open interval $(B, R)$ is contractible if $R$ is not a facial region, and has the homotopy type of a sphere of dimension $n-2-k$ if $R=R(F)$ and $F$ is a face of dimension $k$.

PROOF. The proof is by induction on the rank of $R$. One can check the result directly if the rank of $R$ is 2 . Without loss of generality we can assume that $\bar{B}$ is bounded, as was argued in [Ed, Lemma 1.12].

Let $(B, R)^{+}$be the subposet of $(B, R)$ defined by $(B, R)^{+}=\left\{R^{\prime}: B<R^{\prime}<\right.$ $R$ and $R^{\prime}$ is facial $\}$. Let $j:(B, R)^{+} \rightarrow(B, R)$ be the inclusion map. The fiber $j^{-1}\left((B, R)_{\leq R^{\prime}}\right)$ is contractible because of a greatest element if $R^{\prime}$ is facial, and is contractible by inductive hypothesis if $R^{\prime}$ is not facial. Hence by Quillen's theorem $[\mathbf{Q u}, \mathbf{p}$. 103] $j$ is a homotopy equivalence, so it suffices to determine the homotopy type of $(B, R)^{+}$. We consider two cases.

If $R$ is a facial region and $R=R(F)$ where $F$ is of dimension $k$, then by [Ed, Lemma 1.3] we have that $(B, R)^{+}$is dually isomorphic to the interval $(F, \hat{1})$ in $\hat{\mathcal{F}}$. And $(F, \hat{1})$ triangulates a sphere of dimension $n-2-k$, since $\mathcal{F}$ triangulates a sphere of dimension $n-1$.

Now suppose that $R$ is not a facial region. Let $\tau=S(R) \cap B, C=\left\{F_{H}^{\prime} \in\right.$ $\left.\mathcal{F}^{\prime}: H \in \mathcal{T}\right\}$ and $\mathcal{F}(C)=\left\{F \in \mathcal{F}: \mathcal{F}^{\prime}(F) \subseteq C\right\}$, where $\mathcal{F}^{\prime}(F)=\left\{F^{\prime} \in \mathcal{F}^{\prime}: F \subseteq F^{\prime}\right\}$. Note that $\mathcal{F}(C)$ is a filter in $\mathcal{F}$. It is shown in Case 2 of [Ed, Theorem 1.11] that $(B, R)^{+}$is dually isomorphic to $\mathcal{F}(\mathcal{C})$. Moreover, $\mathcal{F}-\mathcal{F}(\mathcal{C})$ is an ideal generated by a set of facets of $\bar{B}$ which form an initial segment of a recursive coatom ordering of $\hat{\mathcal{F}}$. See $[\mathbf{E d}$, Theorem 1.8]. Hence by Theorem 1.3, $\mathcal{F}(\mathcal{C})$ is contractible and hence so is $(B, R)^{+}$.

Corollary 2.3 [Ed, TheOREM 1.11]. For any region $R$ in $P(\mathcal{H}, B)$,

$$
\mu(B, R)= \begin{cases}(-1)^{n-k} & \text { if } R=R(F) \text { and } \operatorname{dim} F=k, \\ 0 & \text { otherwise. }\end{cases}
$$

Proof. This follows directly from Theorem 2.2 , since $\mu(B, R)$ is the reduced Euler characteristic of $(B, R)$.

COROLLARY 2.4. If $R_{1}<R_{2}$ in $P(\mathcal{H}, B)$, then $\left(R_{1}, R_{2}\right)$ is either contractible or homotopy equivalent to a sphere.

ProOF. It is easy to show that the interval $\left(R_{1}, R_{2}\right)$ in $P(H, B)$ is isomorphic to the interval $\left(R_{1}, R_{2}\right)$ in $P\left(\mathcal{H}, R_{1}\right)$. Then apply Theorem 2.2 .

COROLlaRY 2.5. If $B$ is bounded, then $P(\not, B)-\{B\}$ is homotopy equivalent to a sphere of dimension $n-1$.

PROOF. The order-reversing injection $R: \mathcal{F} \rightarrow P(H, B)-\{B\}$ has contractible fibers by the proof of Theorem 2.2, hence it induces homotopy equivalence. And $\mathcal{F}$ triangulates a sphere of dimension $n-1$.

In [Co] Cordovil generalizes hyperplane posets to a poset on the acyclic orientations of an oriented matroid and proves the generalization of Corollary 2.3 in that context. We conjecture that Theorem 2.2 can similarly be generalized. 


\section{REFERENCES}

[Bj1] A. Björner, Shellable and Cohen-Macaulay partially ordered sets, Trans. Amer. Math. Soc. 260 (1980), 159-183.

[Bj2] _ Orderings of Coxeter groups, Contemporary Mathematics, vol. 34 (edited by C. Greene), Amer. Math. Soc., Providence, R. I., 1984.

[BM] H. Brugesser and P. Mani, Shellable decompositions of cells and spheres, Math. Scand. 29 (1971), 197-205.

[BW] A. Björner and M. Wachs, On lexicographically shellable posets, Trans. Amer. Math. Soc. 277 (1983), 323-341.

[Co] R. Cordovil, A combinatorial perspective on the non-Radon partition, J. Combin. Theory Ser. A (to appear).

[Ed] P. H. Edelman, A partial order on the regions of $\mathbf{R}^{n}$ dissected by hyperplanes, Trans. Amer. Math. Soc. 283 (1984), 617-631.

[Pr] J. S. Provan, Decompositions, shellings, and diameters of simplicial complexes and convex polyhedra, Cornell Univ. School of Operations Research and Industrial Engineering, Technical Report No. $354,1977$.

[Qu] D. Quillen, Homotopy properties of the poset of nontrivial p-subgroups of a group, Adv. in Math. 28 (1978), 101-128.

[Wa] J. W. Walker, Homotopy type and Euler characteristic of partially ordered sets, European J. Combin. 2 (1981), 373-384.

Department of Mathematics, University of Pennsylvania, Philadelphia, PENNSYLVANIA 19104

Department of Mathematics, University of South Carolina, Columbia, SOUTH CAROLINA 29208 